\title{
EFFECTS OF OVERWEIGHT, DIABETES AND SMOKING ON EARLY FETAL GROWTH
}

\author{
J.H. Grundt ${ }^{1}$, J.O. Nakling ${ }^{2}$, T. Markestad ${ }^{3,4}$
}

${ }^{1}$ Pediatrics, ${ }^{2}$ Obstetrics \& Gynecology, ${ }^{3}$ Research Dept., Sykehuset Innlandet Trust, Lillehammer, ${ }^{4}$ Dept. of Clinical Medicine, University of Bergen, Bergen, Norway

Background and aims: Environmental influences in utero may increase risk of later overweight and obesity. A possible mechanism may be metabolic programming associated with early fetal growth. A difference (EDD-diff) between estimated date of delivery based on last menstrual period (EDD-LMP) and ultrasound around 18 weeks' pregnancy (EDD-US) may indicate restricted or accelerated early fetal growth.

Methods: In a population based pregnancy data base 18271 liveborn singleton term babies without malformations had reliable EDD-LMP estimates and EDD-US determination and had not used oral contraceptives during the last 3 months before pregnancy. Groups were compared using t-tests and the significance of various factors on EDD-diff was assessed using multiple linear regression analysis.

Results: Compared to EDD-LMP EDD-US resulted in significant delays in EDD in smokers vs. nonsmokers $(0.39$ days, $95 \%$ CI $0.14-0.63)$, in obese $(1.71$, CI $1.31-2.12)$, overweight $(0.62$, CI $0.31-0.92)$ and underweight $(0.96$, CI 0.17 - 1.74) vs. normal weight women, and in diabetics vs. non-diabetics $(2.16$, CI 1.21 - 3.11). In the regression analysis $(n=8128)$ maternal age, height, parity, pre-pregnancy BMI and sex of the fetus explained $5 \%$ of the difference in EDD-diff; overweight and obesity (0.102 days/BMI-unit), but not smoking made significant contribution.

Conclusions: Overweight, underweight and diabetes, but not smoking in pregnancy resulted in minor early fetal growth restriction. The difference did not affect determination of EDD, but we speculate that it may be of significance as factors affecting risk of later overweight in the offspring. 